\title{
Programming Parameter
}

National Cancer Institute

\section{Source}

National Cancer Institute. Programming Parameter. NCI Thesaurus. Code C48913.

A value that is passed to a routine. 